\title{
Glioma Specific Extracellular Missense Mutations in the First Cysteine Rich Region of Epidermal Growth Factor Receptor (EGFR) Initiate Ligand Independent Activation
}

\section{Susie I. Ymer ${ }^{1, \dagger}$, Sameer A. Greenall ${ }^{1, \dagger}{ }^{1,}$, Anna Cvrljevic ${ }^{1}$, Diana X. Cao ${ }^{2}$, Jacqui F. Donoghue ${ }^{1}$,} V. Chandana Epa ${ }^{3}$, Andrew M. Scott ${ }^{2}$, Timothy E. Adams ${ }^{3}$ and Terrance G. Johns ${ }^{1}$ *

1 Oncogenic Signaling Laboratory, Monash Institute of Medical Research, Monash University, Clayton, VIC 3168, Australia; E-Mails: ymersi@gmail.com (S.I.Y.), sameer.greenall@csiro.au (S.A.G.), acurljev@btk.fi (A.G.)

2 Tumor Targeting Laboratory, Ludwig Institute for Cancer Research, Heidelberg, VIC 3084, Australia; E-Mails: diana.cao@ludwig.edu.au (D.X.C.), andrew.scott@ludwig.edu.au (A.M.S.)

3 CSIRO Division of Materials Science and Engineering, Parkville, VIC 3052, Australia; E-Mails: vidana.epa@csiro.au (V.C.E.); tim.adams@csiro.au (T.E.A.)

$\dagger$ These authors contributed equally to this work.

* Current address: CSIRO Division of Materials Science and Engineering, Parkville, VIC 3052, Australia.

* Author to whom correspondence should be addressed; E-Mail: Terry.Johns@ @ed.monash.edu.au Tel: +613-9594-7247; Fax: +613-9594-7114.

Received: 25 February 2011; in revised form: 29 March 2011 / Accepted: 7 April 2011 / Published: 18 April 2011

\begin{abstract}
The epidermal growth factor receptor (EGFR) is overexpressed or mutated in glioma. Recently, a series of missense mutations in the extracellular domain (ECD) of EGFR were reported in glioma patients. Some of these mutations clustered within a cysteine-rich region of the EGFR targeted by the therapeutic antibody mAb806. This region is only exposed when EGFR activates and appears to locally misfold during activation. We expressed two of these mutations (R324L and E330K) in NR6 mouse fibroblasts, as they do not express any EGFR-related receptors. Both mutants were autophosphorylated in the absence of ligand and enhanced cell survival and anchorage-independent and xenograft growth. The ECD truncation that produces the de2-7EGFR (or EGFRvIII), the most common EGFR mutation in glioma, generates a free
\end{abstract}


cysteine in this same region. Using a technique optimized for detecting disulfide-bonded dimers, we definitively demonstrated that the de2-7EGFR is robustly dimerized and that ablation of the free cysteine prevents dimerization and activation. Modeling of the R324L mutation suggests it may cause transient breaking of disulfide bonds, leading to similar disulfide-bonded dimers as seen for the de2-7EGFR. These ECD mutations confirm that the cysteine-rich region of EGFR around the mAb806 epitope has a significant role in receptor activation.

Keywords: EGFR; de2-7EGFR; extracellular domain mutation; autoactivation; dimerization; disulfide bond; free cysteine

\section{Introduction}

The epidermal growth factor receptor (EGFR) belongs to the ErbB family of receptor tyrosine kinases (RTKs) that also include ErbB2, ErbB3 and ErbB4 [1]. EGFR can bind at least seven ligands, of which the most widely studied is epidermal growth factor (EGF) [1]. Regulated activation of the EGFR requires high affinity ligand binding and involves homo- and/or hetero-dimerization with other ErbB family members [2]. Dimerization is essential for activation of its intrinsic kinase activity and subsequent autophosphorylation [3-6]. These events activate a number of different downstream signaling pathways that regulate multiple cellular processes such as proliferation, differentiation and development. The EGFR is commonly overexpressed or mutated in many cancer types and its presence promotes tumor progression and survival.

In glioblastoma multiforme (glioma), a highly malignant and lethal neoplasm of the brain, amplification of the EGFR gene leads to overexpression of the receptor and is associated with a number of mutations. The most common EGFR mutation in glioma is the de2-7EGFR, a 267 amino acid deletion of the extracellular domain (ECD) [7]. It is constitutively active, unable to bind known ligand, cancer specific, and is not detectable in normal tissue [8]. The clinical importance of de2-7EGFR is evident as its expression is closely linked to increased tumor aggression, invasion and poor prognosis for survival [7].

The antibody mAb806 which was raised against the de2-7EGFR, also binds the full length EGFR but only when it is activated through overexpression, mutation or autocrine activity [9,10]. Since the EGFR is not constitutively active in normal tissue, the mAb806 is cancer-specific. Epitope mapping has shown that the mAb806 epitope is located in short cysteine loop of the EGFR (amino acids 311-326) [11].

Recently, several novel glioma-specific missense mutations in the ECD of the EGFR were identified [12]. Two of these mutations, R324L and E330K, are located within or close to the region of the mAb806 binding site. Importantly, a similar corresponding region in ErbB2 has also been found to be mutated [13]. Given the location of these mutations and their proximity to the mAb806 binding site, we sought to understand the function of these missense mutations in NR6 cells as these do not express any ErbB family members. Interestingly, the N-terminus of the de2-7EGFR ECD also starts in this region and contains a free cysteine adjacent to the mAb806 epitope. Using the de2-7EGFR as a model we examined the role of cysteine residues in this region with respect to mutant EGFR activation. 


\section{Results}

\subsection{Expression of the ECD Mutations}

The expression of two EGFR ECD mutants (R324L and E330K) was examined in a cell-free system and NR6 cells. The R324L mutation is located within the disulfide loop containing the mAb806 epitope and the E330K mutation is located within an adjacent disulfide loop. Characterization of the A289V has been reported previously [12] and was included as a control in several experiments.

The cell-free translation of mutant EGFR demonstrated that Mw and expression levels of the proteins were similar to the wtEGFR, indicating that the mutations did not affect translation efficiency (Figure 1A). Both the wtEGFR and mutant EGFR were expressed on the cell surface of NR6 cells as determined by Fluorescence Activated Cell Sorting (FACS) (Figure 1B). Since the mAb528 antibody is highly conformation dependant, its ability to recognize the mutants suggests that they are folded correctly. The mAb806 recognized a portion of the overexpressed wtEGFR as expected (Figure 1B), but a greater shift was evident for wtEGFR and A289V than the R324L or E330K mutants, suggesting that the latter two mutations may affect the binding of the mAb806. This was also observed in our Western analyses (Figure 1C) where mAb806 displayed reduced binding especially to the R324L mutation relative to wtEGFR (upper panel). Binding of a C-terminal EGFR antibody was unchanged (lower panel) when comparing the fully glycosylated upper EGFR band. Thus the R324L and E330K mutations are expressed on the cell surface and have a minor, but reproducible, impact on mAb806 binding.

\subsection{ECD EGFR Mutations Are Constitutively Active and Retain Response to Ligand}

To examine if the EGFR mutants were constitutively activated or could respond to ligand, Whole cell lysates (WCL) from transgenic NR6 cells were blotted with a panel of pY EGFR antibodies (Figure 2). Basal phosphorylation of the EGFR (Figures 2A and 2B) was enhanced in the absence of ligand stimulation for both the R324L and E330K mutants slightly at Y1173 (2 fold and 5 fold increases when corrected for total EGFR, respectively) and dramatically at Y992 (3 fold and 10 fold increases, respectively) relative to wtEGFR. Examination of the Y1086 and Y1148 sites also showed enhanced basal phosphorylation for the mutants, particularly R324L, relative to wtEGFR (Figure 2C). However, Y845 basal phosphorylation, a target of Src phosphorylation and not autophosphorylation, was only increased slightly (Figure 2C). EGF stimulation resulted in increased phosphorylation at Y1173 and Y992, the two sites examined, for both R324L and E330K (Figures 2A and 2B). Thus the R324L and E330K mutants are constitutively active and respond to ligand.

\subsection{Downstream Signaling by EGFR Mutations}

We determined if the EGFR mutations affected the activation of the extracellular signal-regulated kinase (ERK) 1/2 and Akt. Although ERK was phosphorylated in response to EGF stimulation (data not shown), basal levels of pERK were significantly decreased for both the R324L and E330K mutants compared to wtEGFR $(P<0.01$ for both; Figure 3A). Basal pAkt:total Akt ratios were similar for wtEGFR and mutant EGFR (Figure 3B). These results suggest that these constitutively active EGFR mutants down-regulate pERK1/2 but do not activate Akt. 
Figure 1. Biochemical characterization of mutant epidermal growth factor receptor (EGFR). (A). wtEGFR and mutant EGFR were translated into ${ }^{35} \mathrm{~S}$-cysteine labelled proteins in a cell free expression system and labeled proteins detected by autoradiography following SDS-PAGE; (B). NR6 cells expressing wtEGFR or mutant EGFR were stained with secondary antibody alone (green), isotype control antibody (orange), mAb528 (red) or mAb806 (blue) and subjected to Fluorescence Activated Cell Sorting (FACS) analysis. The representative profiles for each cell line are shown; $(\mathbf{C})$. wtEGFR or mutant EGFR cells were grown under serum free conditions, lysed and IP'd using mAb528. Western blot analysis of total EGFR using a mAb806 probe (upper panel) or a C-terminal EGFR probe (lower panel) are shown. A431 cells that overexpress the EGFR were included as a positive control.
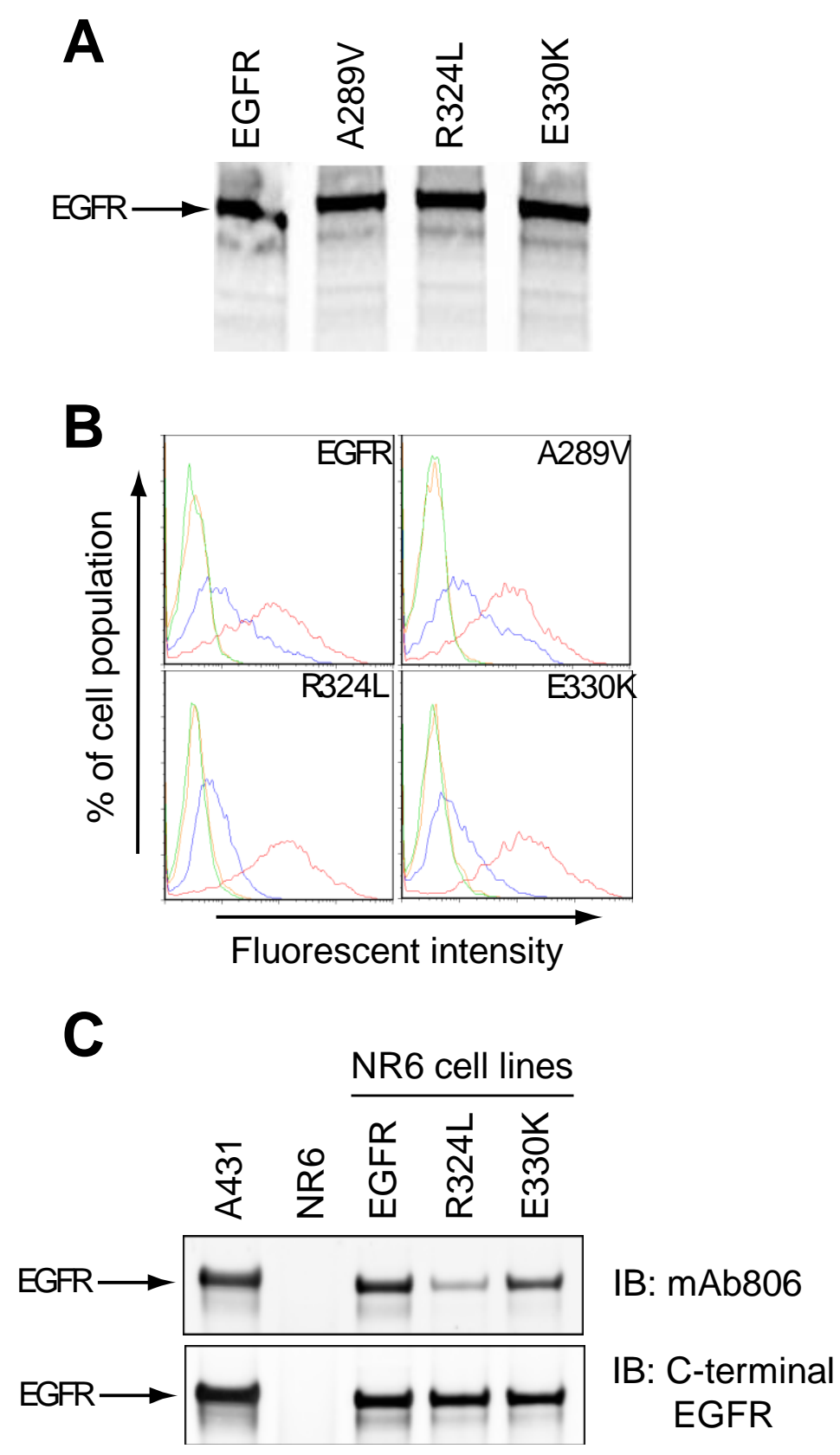
Figure 2. Activation of the EGFR mutants. Whole cell lysates (WCL) from NR6 cells expressing wtEGFR or the R324L and E330K mutants were analyzed by Western blot at multiple phosphorylation sites without or with EGF stimulation. (A). pY1173 analysis (upper panel) and C-terminal EGFR (lower panel). A431 cells that overexpress the EGFR were included as a positive control; (B). pY992 blot (upper panel) and C-terminal EGFR (lower panel); (C). Basal phosphorylation status of pY845 (left), pY1086 (middle) and pY1148 (right) blots and their corresponding actin loading controls. In all cases representative results from multiple repeats are shown.
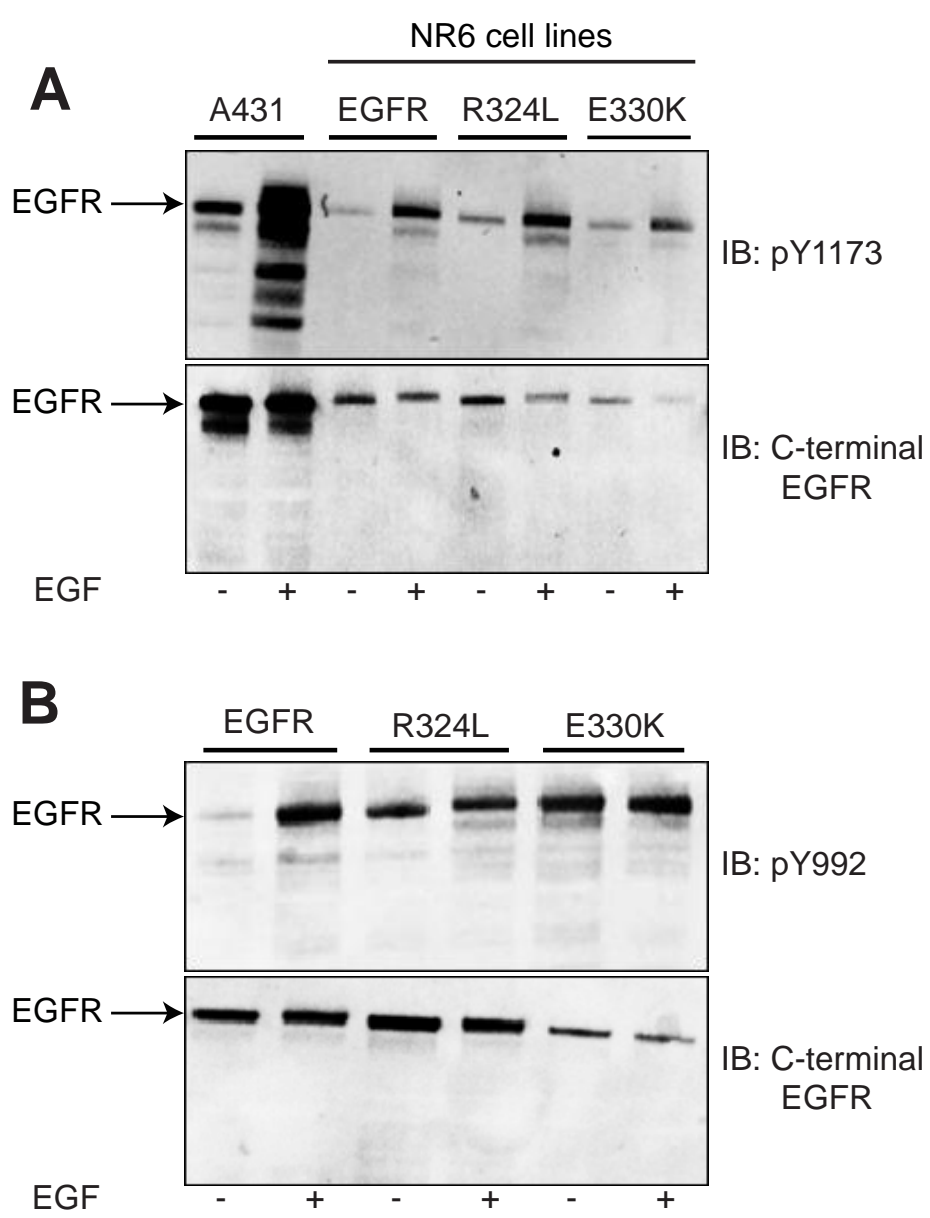

C

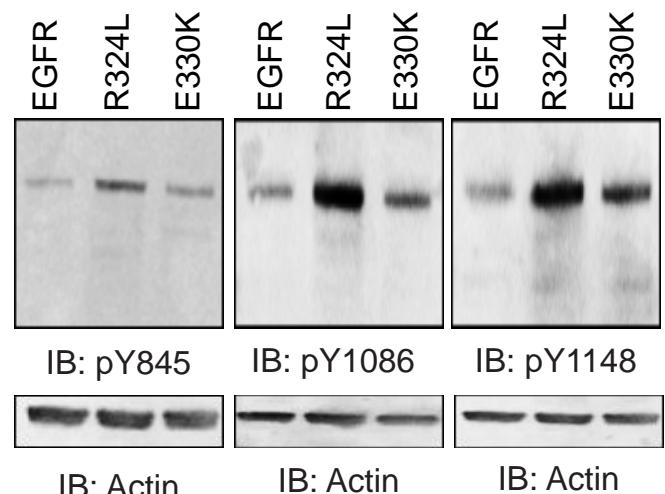


Figure 3. Activation of downstream pathways by mutant EGFR. Transgenic NR6 cells expressing wtEGFR, R324L and E330K were plated under serum free conditions and then lysed and tested by Bioplex for (A) pERK1/2 and (B) pAkt levels. Data is presented as mean fluorescent intensity \pm S.E. or as a ratio of $\mathrm{p}$-Akt to t-Akt \pm S.E. of three independent replicates corrected for total Akt protein.

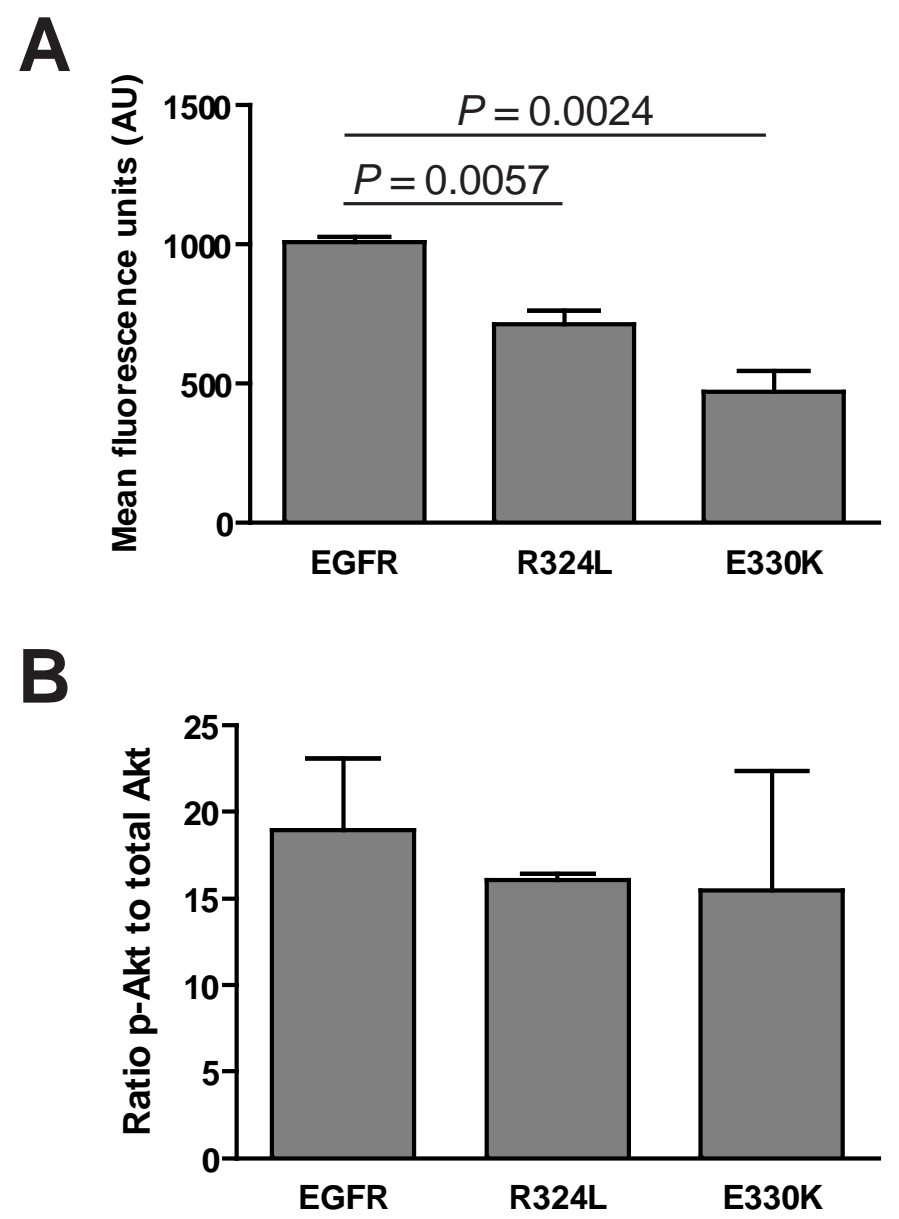

\subsection{EGFR Mutations Bestow an In Vitro Survival Advantage to NR6 Cells}

The survival of NR6 cells expressing the EGFR mutants was examined under serum-free and low serum conditions at various time points to determine any in vitro survival advantages. The NR6 cells expressing R324L showed enhanced proliferation within the first $24 \mathrm{~h}$ of culture (Figure 4A). Also, while the number of wtEGFR and A289V NR6 cells decreased substantially over $48 \mathrm{~h}$ in serum free conditions, the number of NR6 cells expressing either R324L or E330K remained steady (Figure 4A), indicating a retention of cell viability. When grown in $2 \%$ fetal bovine serum (FBS) over $96 \mathrm{~h}$ (Figure 4B), the cell numbers for the R324L and E330K mutants continued to increase and were significantly higher than the wtEGFR and A289V cells, which began to die, at the $96 \mathrm{~h}$ time-point. These data show that the R324L and E330K also provide a survival advantage to cells in low serum conditions. 
Figure 4. The R324L and E330K mutants show increased cell survival under serum free and 2\% FBS conditions. Transgenic cells were cultured under (A) serum free conditions for $48 \mathrm{~h}$ and (B) in media containing 2\% FBS for $96 \mathrm{~h}$. Cells were tested for proliferation at various time points using the MTS assay. The data shown is the percentage of cell growth at each time point compared to the $0 \mathrm{~h}$ control \pm S.E.
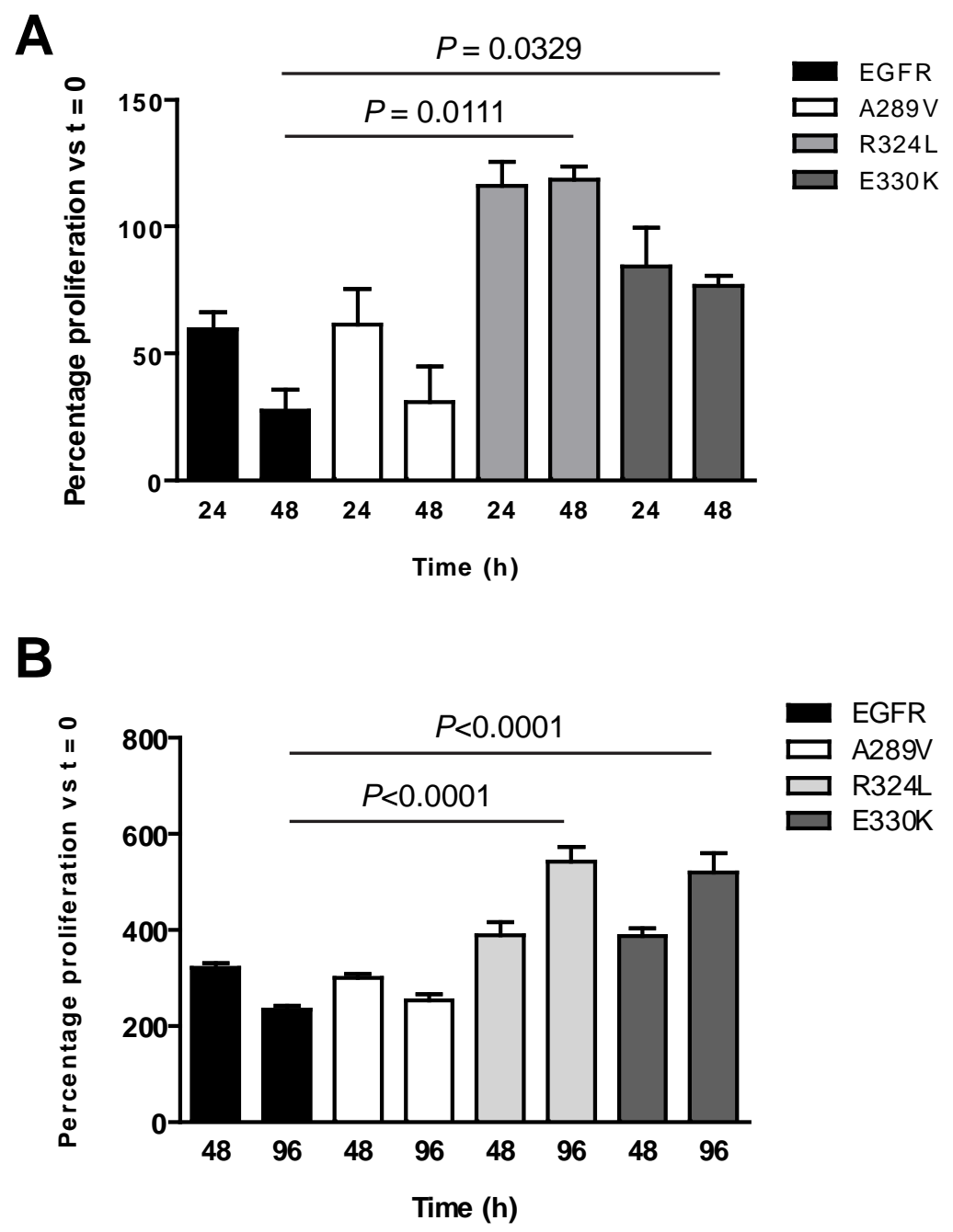

The ability of the transgenic NR6 cells to form colonies in anchorage-free conditions was examined (Figure 5). All of the cells tested formed colonies, with the R324L mutant forming significantly more colonies than the other cell lines $(P=0.009$; Figure 5$)$. The E330K mutant did not form significantly more colonies than wtEGFR-expressing cells. When the data was grouped into the percentage of colonies greater than $120 \mu \mathrm{m}$ (Figure 5B) and $150 \mu \mathrm{m}$ (Figure 5C), it was clear that both the mutant EGFR-expressing cells formed colonies that were significantly larger in size than wtEGFR. Thus, the ECD EGFR mutations significantly enhanced in vitro survival in serum free conditions and soft agarose when compared with wtEGFR.

\subsection{EGFR Mutations Increase Tumorgenicity of NR6 cells In Vivo}

Transgenic NR6 cells expressing wtEGFR, R324L or E330K were injected s.c. into BALB/c nude mice and tumor development was followed for 50 days. Expression of the EGFR mutations in NR6 
cells caused an accelerated growth rate over time compared to the wtEGFR (Figure 6A). The R324L and E330K tumors at day 40 were 4-5 times bigger than that observed for wtEGFR tumors $\left(1.15-1.5 \mathrm{~cm}^{2}\right.$ vs. $0.25 \mathrm{~cm}^{2}$ for the mutant EGFR and wtEGFR respectively). Examination of the tumor appearance after surgical resection revealed that the mutant EGFR tumors were bulbous and heavily vascularized, whereas the wtEGFR tumors were flat, pale and possessed little vascularization (Figure 6B). These results show that the R324L and E330K mutants conferred a significant growth advantage and enhanced tumorgenicity of NR6 cells in vivo.

Figure 5. The R324L and E330K mutants demonstrate enhanced transforming activity in anchorage independent growth assays. Transgenic NR6 cells were plated in an agarose matrix for 20 days and stained with MTT. (A) Colonies were counted and data graphed as the total colonies per well \pm S.E. A random 10-20\% sampling of the total numbers was analyzed for the percentage of cells (B) over $120 \mu \mathrm{m}$ or $(\mathbf{C})$ over $150 \mu \mathrm{m}$ in size \pm S.E.
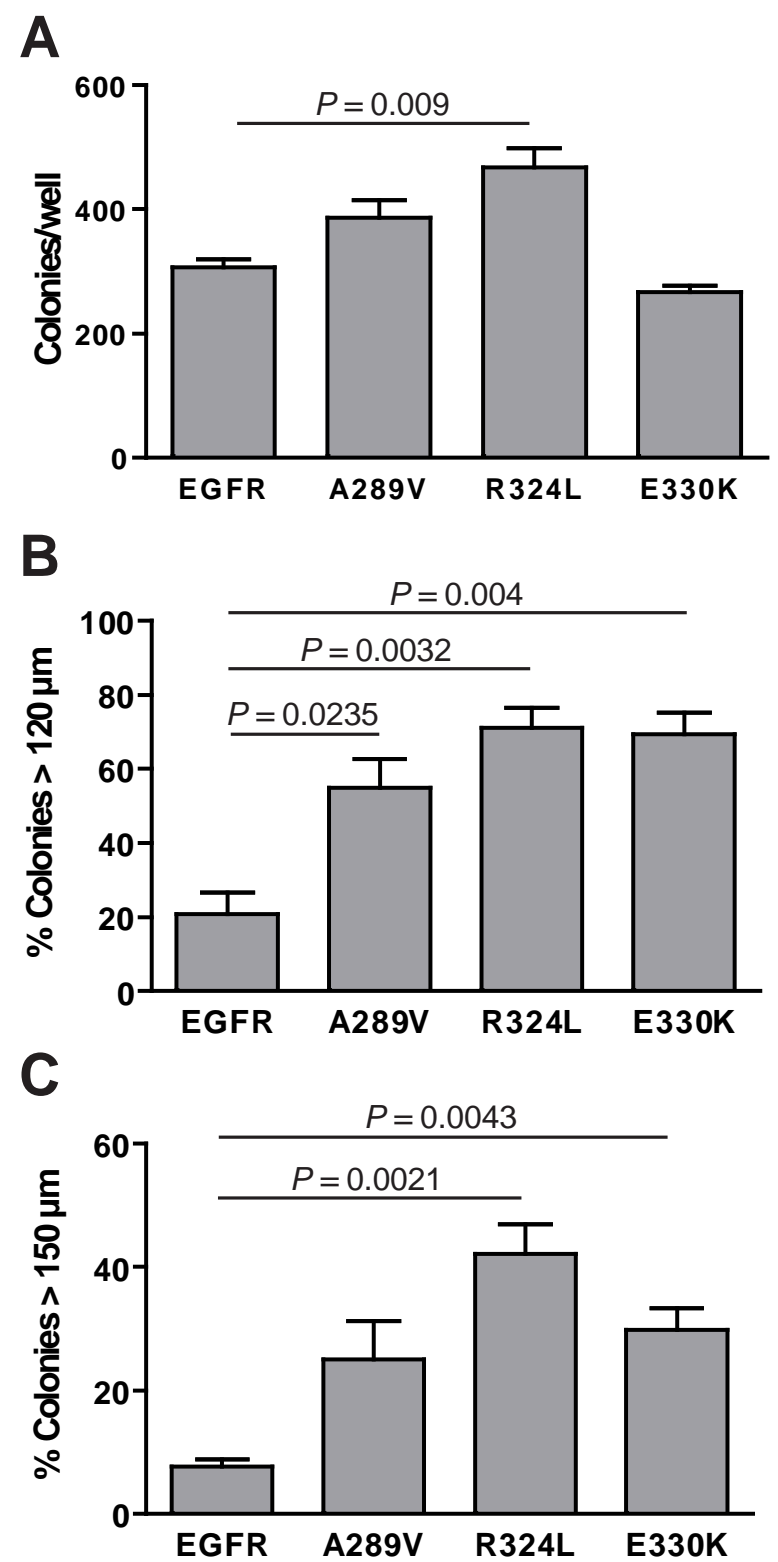
Figure 6. EGFR mutants promote in vivo tumor growth. Nude mice were injected subcutaneously into both flanks with transgenic NR6 cells containing wtEGFR, R324L or E330K. (A) Growth curves for NR6 cells expressing wtEGFR or R324L or E330K mutants. Data is presented as mean tumor volume \pm S.E; (B) Gross tumors surgically resected for E330K, R324L and wtEGFR after 40 days.

A

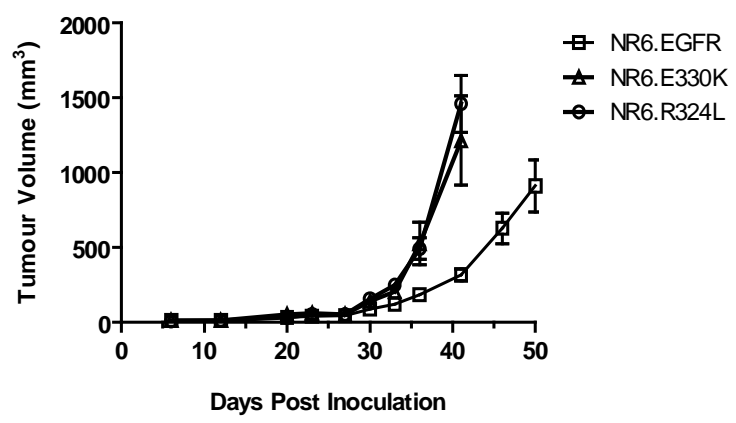

B

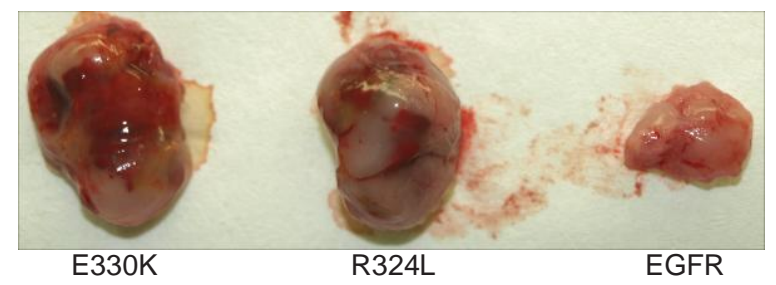

2.6. de2-7EGFR Undergoes Reduction-Sensitive Dimerization Utilizing a Free N-Terminal Cysteine

The de2-7EGFR truncation breaks the EGFR cysteine pairing of C295-C307 and removes the C295 residue, creating an unpaired cysteine at $\mathrm{C} 307$ (identified by us here as $\mathrm{C} 16$ in the mature de2-7EGFR sequence). This cysteine maps to the same region as our missense mutations. In addition, it is possible that this free cysteine could lead to dimerization and activation of de2-7EGFR via formation of an intermolecular disulfide bond. To investigate this, we mutated the $\mathrm{C} 16$ to serine $(\mathrm{C} 16 \mathrm{~S})$ in the de2-7EGFR. Both the de2-7EGFR and the C16S were successfully expressed in U87MG cells (Figure 7). Under non-reducing conditions (upper left panel, Figure 7A), a de2-7EGFR dimer was clearly observed but was virtually absent in the C16S mutant even when the blots were overexposed (left panel, Suppl. Figure 1) proving that de2-7EGFR dimerization requires this free cysteine. Quantification of the dimer: Monomer densitometry ratios for total protein (lower left graph, Figure 7A) showed the de2-7EGFR dimer formed a high proportion of the total species $(\sim 25 \%)$. As expected, the dimer disappeared in the reducing mAb806 blot (left panel, Figure 7B). When non-reducing blots were probed with the pY1173 EGFR antibody, the de2-7EGFR dimer was strongly phosphorylated whilst no phosphorylated dimer was detectable for C16S (upper middle panel, Figure 7A). Densitometry analyses confirmed that the de-7EGFR dimer was the active form of the receptor as it was 4.1 times more intense than the monomer (lower right graph, Figure 7A). Reduction of the samples and probing for pY1173 showed that the overall phosphorylation of the C16S mutant was markedly decreased compared to the unmodified de2-7EGFR (middle panel, Figure 7B). These data demonstrate that the free cysteine created as a consequence of the de2-7EGFR mutation is critical for the formation and stabilization of the active de2-7EGFR dimer. 
Figure 7. de2-7EGFR forms reduction-sensitive homodimers in vitro through its free cysteine residue. Cells were lysed and equal protein probed by Western blot under non-reducing (A) and reducing (B) conditions for total EGFR/de2-7EGFR by mAb806 blotting (left hand panels) and pY1173 (middle panels). A pan actin blot was used as the loading control (right hand panels). Graphed data of dimer:monomer densitometry ratios \pm S.E. for non-reducing mAb806 (lower left graph) and pY1173 (lower right graph) are depicted under each blot in (A). U87MG parental cells (U87MG) and U87MG overexpressing the wtEGFR (EGFR) were included as control for the wtEGFR on the blots. In all cases representative results from multiple repeats are shown $(\mathbf{C}) \mathrm{C} 16 \mathrm{~S}$ is less tumorigenic than unmodified de2-7EGFR. Nude mice were injected subcutaneously into both flanks with transgenic U87MG cells expressing either unmodified de2-7EGFR or the C16S variant. Kaplan-Meier survival curves were then determined based on a tumor volume of $1000 \mathrm{~mm}^{3}$.

A

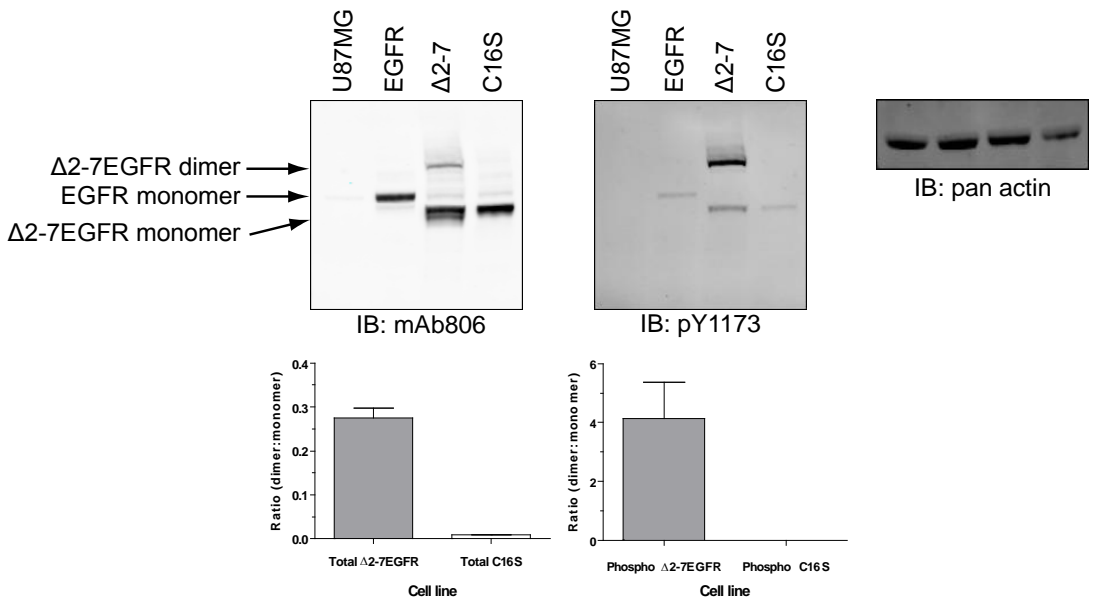

B

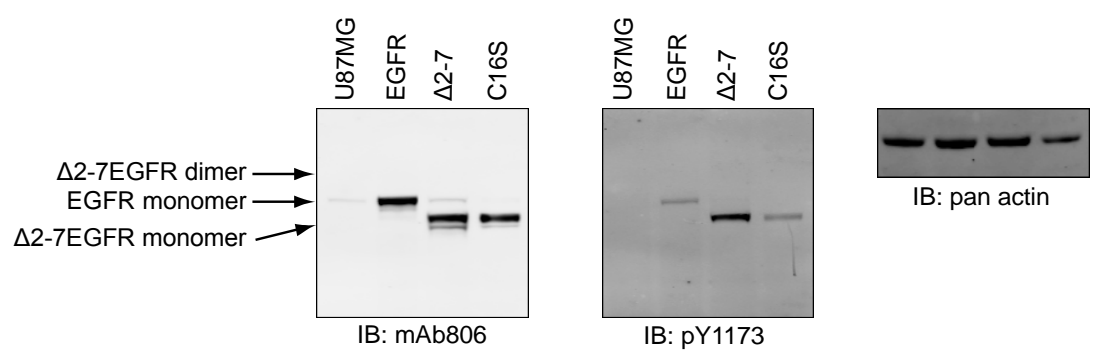

C

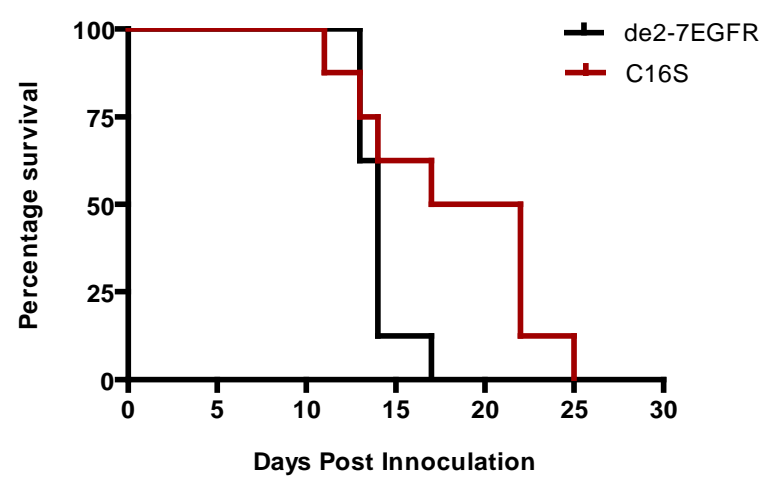




\subsection{The C16S Modification Displays a Reduced Tumorigenicity In Vivo}

In order to see if the ablation of the free cysteine in de2-7EGFR has any in vivo consequences, U87MG cells expressing unmodified de2-7EGFR or the C16S variant were injected s.c. into BALB/c nude mice and tumor development followed. The survival of each population was then compared by Kaplan-Meier survival analysis (Figure 7C). Animals injected with C16S variant survived significantly longer $(P<0.05)$ than those injected with unmodified de2-7EGFR, demonstrating that the reduction observed in in vitro activity translates into a reduced tumorigenicity in vivo.

\section{Discussion}

Glioma specific single point missense mutations in the cysteine rich region of EGFR near the mAb806 epitope lead to autoactivation and enhanced tumorigenicity. There were pronounced differences with respect to the tyrosine phosphorylation pattern seen for each mutant and neither R324L nor E330K strongly activated pY1173, previously identified as the key phosphorylation site for activity of the de2-7EGFR [14]. This suggests that clonal selection within the tumor might result in EGFR mutations with differing signaling properties. The preponderance of ECD mutations in glioma, in contrast to the kinase mutations that dominate lung cancer, further supports the idea that mutations arise in a context specific manner. Both mutants, but especially E330K, showed increased phosphorylation at Y992. Phosphorylation at this site reduces ligand-induced receptor endocytosis, increasing the lifetime of the activated receptor in the plasma membrane and therefore enhancing signaling capacity [15]. Recently, an Akt-independent PLC $\gamma$-PKC $\alpha$-mTOR pathway was identified in glioma [16]. It is possible that our mutations preferentially activate this pathway and may partially explain the lack of Akt activation. The decrease in pERK mediated by the R324L and E330K mutant is consistent with recent findings showing pERK is negatively correlated with de2-7EGFR [17].

Various reports suggest that the de2-7EGFR only forms transient homodimers [14,18,19]. An early publication showing robust dimerization of de2-7EGFR has not been reproduced [20] and a second paper reporting significant dimerization used non-physiological conditions including a lengthy purification [21]. However, de2-7EGFR homodimers must exist as auto-phosphorylation of the EGFR always occurs in trans through the formation of an asymmetric dimer [22]. Indeed, we used differentially tagged de2-7EGFR molecules to demonstrate this transient interaction [18]. Using the drug iodoacetamide during lysis to protect disulfide bonds, we could reproducibly show significant levels of dimerized de2-7EGFR. Importantly, detection of the de2-7EGFR dimer in our system was totally dependent on the presence of the free cysteine at position 16. The previous confusion about the presence or absence of de2-7EGFR dimers seems to be caused by the use of differing techniques. Detection of these dimers requires the addition of reagents that protect the intermolecular disulfide bond as it appears extremely labile during cell lysis. Finally, the intense phosphorylation of the de2-7EGFR dimer, especially when the phosphorylated receptor is compared to total receptor (Figure 7), clearly demonstrates that this is the active conformation.

Unfortunately, while we were able to show that the missense mutations were present as dimers using the techniques described here (data not shown), we were unable to definitively determine their nature because of their low abundance and the presence of alternative inactive dimers as previously reported [23]. Also, the ability to mutate the exact cysteine residue in the de2-7EGFR made the definitive experiments 
relatively straight-forward in this receptor. Obviously this approach is not possible in full-length receptors. We have postulated that the region around mAb806 must be locally misfolded during receptor activation [10]. As noted, the R324L mutation is found within the mAb806 epitope and the E330K mutation is in the adjacent cysteine loop (Figure 8A). Our results show that these mutations cause conformational changes that enhance receptor activation in the absence of ligand. The R324L mutation abolishes two salt bridges with adjacent E317 and E319 residues. These salt bridges appear to stabilize the C311-C326 disulfide loop (Figure 8B). Their removal should increase the flexibility and dynamic behavior of the loop and place strain on the C311-C326 disulfide bond. This would make the bond more susceptible to breaking and possibly prevent its formation, leaving a free cysteine for intermolecular bonding as for the de2-7EGFR. Indeed, the cysteine at 311 is only a few amino acids away from the free cysteine in de2-7 EGFR which is cysteine 307 (or C16 in the de2-7EGFR). Molecular modeling has suggested that a G336R ECD mutation found in zebra fish EGFR also disrupts a nearby disulphide bond resulting in the formation of disulphide linked homodimers [24]. Taken together these results suggest a general mechanism of activation, whereby mutations in this cysteine rich region lead to disruption of disulfide bonds which become available for stabilizing dimer interactions.

Figure 8. (A). Alignment of amino acids 295-337 of the immature EGFR protein with amino acids 1-46 of the mature de2-7EGFR protein. The disulfide bonds (black lines), mAb806 epitope and position of two EGFR ECD mutations studied in this paper (red) are shown. A 267 amino acid truncation removes the C295 from the EGFR structure and results in a free $\mathrm{C} 16$ residue in the mature de2-7EGFR protein (pink) as well as a unique 6 amino acid N-terminal sequence (green); (B). Three dimensional structural model of module 7 incorporating the R324L mutation. R324 lies within the module 7 loop spanning amino acids 311-326 in Domain II of EGFR. The R324L mutation will abolish salt bridges (dashed lines) with E317 and E319 which stabilize the loop, leading to greater loop flexibility and significant strain on the C311-C326 bond (yellow). Analysis utilized the structure of ligand bound EGFR extracellular domain described previously [3]. The figure was prepared with PyMol v.1.2r2 software (Schrodinger, LLC).

A

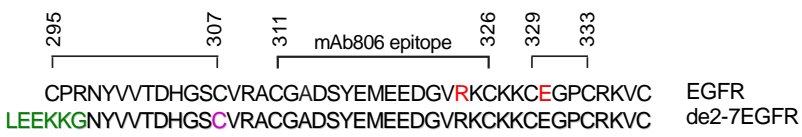

B

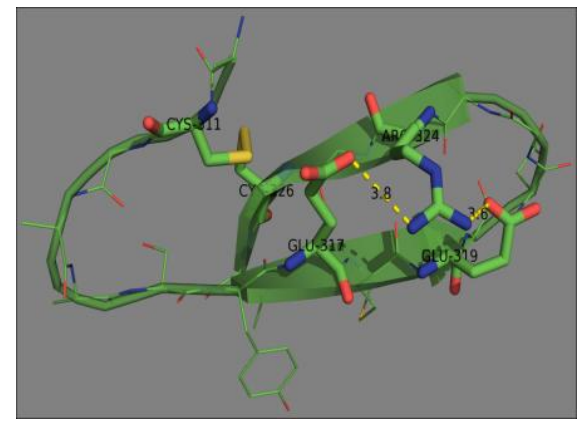


Mutations in other RTKs have been shown to create free cysteines that mediate receptor dimerization. The ECD mutations W290G or T341P within FGFR-2, which are directly linked to the initiation of Crouzon and Pfeiffer syndromes, release a free cysteine by disrupting a nearby intramolecular disulfide bond, triggering intermolecular bonding, activation and conferring tumorigenic properties in NIH3T3 cells [25]. Other examples of free cysteine-linked activation include the R129C ECD erythropoietin receptor mutation [26,27], C342Y ECD FGFR-2 mutation [28] and the c-RET MEN-2A ECD germline mutation C634R [29]. All of these mutants are dimerized, constitutively active and directly linked to induction of various diseases or cancers.

\section{Experimental}

\subsection{EGFR Numbering}

Through-out the paper we have numbered EGFR based on the initiating methionine, as per the original mutation paper. However, numbering of phosphospecific antibodies is based on the mature EGFR as per common use. The difference between the two numbering formats is 24 amino acids.

\subsection{Antibodies and Reagents}

mAb528 and mAb806 were produced and purified at the biological production facility at the Ludwig Institute for Cancer Research, Melbourne, Australia. The anti-C-terminal EGFR mAb R13 was from BD Biosciences (San Jose, CA). The anti-C-terminal EGFR polyclonal antibody 1005 and the Protein A/G beads were from Santa Cruz Biotechnology (Santa Cruz, CA). All anti-phosphorylated EGFR antibodies (pY845, pY992, pY1068, pY1086, pY1148 and pY1173), as well as the anti-mouse IgG conjugated to Alexa Fluor 680, were from Invitrogen (Carlsbad, CA). The anti-phosphorylated Y1173 EGFR (clone 53A5) and the anti- $\beta$-actin polyclonal were from Cell Signaling (Danvers, MA). The anti-pan actin mAb ACT05 was from ThermoFisher Scientific (Waltham, MA). Horse radish peroxidase (HRP)-conjugated anti-mouse or anti-rabbit secondary antibodies were from Millipore (Billerica, MA). Anti-mouse IgG whole molecule-R-phycoerythrin (PE) conjugated secondary antibody, human recombinant EGF and iodoacetamide were from Sigma Aldrich (St Louis, MO). Anti-rabbit IgG polyclonal antibody conjugated to IRDye800 was from LiCor Biosciences (Lincoln, NE). Site directed QuikChange mutagenesis XL kit was from Stratagene (La Jolla, CA). All primers were synthesized at Sigma Aldrich or at Micromon (Monash University, Clayton).

\subsection{Cell Lines}

The human glioma cell line U87MG (HTB-14) and the human epidermal carcinoma cell line A431 (CRL-1555) were sourced from the American Type Culture Collection (ATCC, USA). The NR6, U87MG. $42-7$ and U87MG.EGFR cell lines have been described by us previously [30]. The NR6.EGFR, NR6.A289V, NR6.R324L and NR6.E330K cell lines were constructed as follows; mutations were introduced into the EGFR ORF, subcloned in pGEM-4Z (Promega), by site-directed mutagenesis using the primer sets detailed in Suppl. Table 1. Next, the mutant EGFR ORF's were cloned into the pBABEpuro retroviral vector and transfected into a viral packaging cell line using Fugene (Roche), with subsequent viral supernatants used to infect NR6 cells. Transfected cells were 
selected in the presence of puromycin $(2 \mu \mathrm{g} / \mathrm{mL})$ and surviving cells FACS sorted for high EGFR expression using mAb528.

The U87MG. $\Delta 2-7_{\mathrm{C} 16 \mathrm{~S}}$ cell line was constructed using primers detailed in Suppl. Table 1 and site-directed mutagenesis to incorporate a Cys-Ser mutation at amino acid position 16 (the equivalent amino acid is C307 in wtEGFR) within the de2-7EGFR ORF also using the pBABEpuro retroviral vector. Cells were FACS sorted with mAb806 to obtain a surface expression level similar to U87MG. $\Delta 2-7$. All cell lines were maintained in DMEM/F12 containing 5\% FBS, 2 mM Glutamax and 100 units of penicillin/streptomycin in the presence of suitable selective drug.

\subsection{In Vitro Cell-Free Protein Expression}

EGFR-related cDNA's were translated into ${ }^{35}$ S-cysteine labelled proteins from pGEM-4Z using the TNT-coupled reticulocyte lysate kit according to manufacturer's protocol (Promega, Madison, WI).

\subsection{Fluorescence Activated Cell Sorting (FACS) Analysis}

$2-3 \times 10^{5}$ cells were incubated with the indicated antibody at $10 \mu \mathrm{g} / \mathrm{mL}$ in $0.1 \%$ human serum albumin/PBS (HSA-PBS) for $1 \mathrm{~h}$ at $4{ }^{\circ} \mathrm{C}$ and then incubated with 1:20 anti-mouse IgG-PE secondary antibody in HSA-PBS at $4{ }^{\circ} \mathrm{C}$ for 30 min. Cells were resuspended in FACS fix solution (PBS; 3.2\% D-glucose; $1 \%$ formaldehyde) and run on a Guava flow cytometer (Millipore).

\subsection{Immunoprecipitation and Western Blotting}

Immunoprecipitation of EGFR from 1-3 $\times 10^{6}$ transgenic NR6 cells was carried out essentially as previously described [30] using $1 \mu \mathrm{g} / \mathrm{ml}$ of mAb528 to capture the EGFR. Bead pellets after washing were resuspended in reducing LDL loading buffer containing $100 \mathrm{mM}$ DTT. Reducing SDS-PAGE and western analysis were essentially as previously described [30].

\subsection{MTS Cell Growth Assays}

Transgenic NR6 cells expressing wtEGFR or mutant EGFR were seeded in triplicate at 5000 cells/well in 96 well plates and allowed to adhere overnight. Cells were washed in serum-free medium after which cells were incubated in serum-free medium at $37{ }^{\circ} \mathrm{C}, 5 \% \mathrm{CO}_{2}$ for $48 \mathrm{~h}$. At $0 \mathrm{~h}, 24 \mathrm{~h}$ and $48 \mathrm{~h}$, MTS was added to the wells. After $3 \mathrm{~h}$ of incubation at $37^{\circ} \mathrm{C}$, the assay was read at $490 \mathrm{~nm}$ using a FLUOstar Optima plate reader (BMG Labtech, Offenburg, Germany).

\subsection{Anchorage-Independent Growth Assay}

Using 6-well plates, a bottom layer of $0.8 \%$ low melting agarose (LMA) was made by combining equal quantities of melted 1.6\% LMA and $2 \times$ media (DMEM-F12; $10 \% \mathrm{FBS} ; 4 \mu \mathrm{g} / \mathrm{mL}$ puromycin). The top layer of $0.4 \%$ LMA was made by combining equal volumes of melted $0.8 \%$ LMA and $2 \times$ media containing the transgenic cells, at 1000 cells/well. Plates were incubated at $37{ }^{\circ} \mathrm{C}, 5 \% \mathrm{CO}_{2}$ overnight, then $500 \mu \mathrm{L} /$ well of media was added and plates incubated for 20 days. Wells were washed twice with PBS and $500 \mu \mathrm{L}$ of $1 \mathrm{mg} / \mathrm{mL}$ MTT was added for $24 \mathrm{~h}$ after colonies photographed and counted. 


\subsection{Dimerization Analysis of de2-7EGFR}

This was conducted using an established protocol [31] with minor modifications. Confluent cells were harvested, washed twice in ice cold PBS ( $\mathrm{pH}$ 8.0) containing $10 \mathrm{mM}$ iodoacetamide and lysed in ice cold RIPA buffer [50 mM Tris ( $\mathrm{pH} 7.5$ ); $150 \mathrm{mM} \mathrm{NaCl} ; 5 \mathrm{mM}$ EDTA; $0.5 \%$ sodium deoxycholate; $0.05 \%$ SDS; $10 \mathrm{mM} \mathrm{NaF}$; protease inhibitor cocktail set 1 (Calbiochem, USA); $200 \mu \mathrm{M} \mathrm{Na}_{3} \mathrm{VO}_{4}$; $10 \mathrm{mM}$ iodoacetamide] for $10 \mathrm{~min}$ on ice. Lysates were sonicated and clarified at $21,100 \times \mathrm{g}$ for $20 \mathrm{~min}$ at $4{ }^{\circ} \mathrm{C}$. Equal protein amounts, as determined by BCA assay (Sigma), were resuspended in either non-reducing Laemmli buffer or reducing Laemmli buffer containing $0.8 \mathrm{M}$ of $\beta$-mercaptoethanol and analyzed by SDS-PAGE and western blotting.

\subsection{Bioplex Assay}

Transgenic NR6 cells plated at $2 \times 10^{5}$ cells/well were serum-starved for $24 \mathrm{~h}$ prior to stimulation with EGF at a final concentration of $100 \mathrm{ng} / \mathrm{mL}$ for $15 \mathrm{~min}$, at $37{ }^{\circ} \mathrm{C}$. Whole cell lysates (WCL) were prepared using Bioplex cell lysis buffer (BioRad, Hercules, CA) and pAkt and pERK1/2 were quantified according to the manufacturer's instructions.

\subsection{In Vivo Xenograft Models}

$2 \times 10^{6}$ transgenic NR6 cells in PBS were injected subcutaneously into both flanks of six week old female BALB/c nude mice. Tumors were monitored and measured as previously outlined [30]. This research project was approved by the Animal Ethics Committee of the Austin Health, Heidelberg, Australia.

\section{Conclusions}

Our previous studies with mAb806 have clearly identified a critical role for the cysteine-rich region around amino acids 311-326 in EGFR activation [10,11]. We now show that glioma-associated missense mutations in this region lead to ligand independent activation of EGFR. Significantly, a recent report identified activating mutations in the same cysteine rich region of ErbB4 in melanoma [32]. These studies and our current observations strongly suggest that local misfolding in this cysteine rich region is important to EGFR activation. Ongoing studies into the nature of this local misfolding, and the potential role of transient disulfide bond cleavage, should be informative with respect to the mechanisms associated with activation of the ErbB family.

\section{Acknowledgements}

This work was supported by the National Health and Medical Council of Australia (Program Grant 280912), the James S. McDonnell Foundation (\#220020173), a research grant from Amgen Inc and the Victorian Government's Operational Infrastructure Support Program. T.G.J is a recipient of a Clinical Fellowship from the Victorian Cancer Agency. 


\section{References}

1. Linggi, B.; Carpenter, G. ErbB receptors: New insights on mechanisms and biology. Tr. Cell Biol. 2006, 16, 649-656.

2. Jorissen, R.N.; Walker, F.; Pouliot, N.; Garrett, T.P.; Ward, C.W.; Burgess, A.W. Epidermal growth factor receptor: Mechanisms of activation and signalling. Exp. Cell Res. 2003, 284, 31-53.

3. Garrett, T.P.; McKern, N.M.; Lou, M.; Elleman, T.C.; Adams, T.E.; Lovrecz, G.O.; Zhu, H.J.; Walker, F.; Frenkel, M.J.; Hoyne, P.A.; et al. Crystal structure of a truncated epidermal growth factor receptor extracellular domain bound to transforming growth factor alpha. Cell 2002, 110, 763-773.

4. Ogiso, H.; Ishitani, R.; Nureki, O.; Fukai, S.; Yamanaka, M.; Kim, J.H.; Saito, K.; Sakamoto, A.; Inoue, M.; Shirouzu, M.; Yokoyama, S. Crystal structure of the complex of human epidermal growth factor and receptor extracellular domains. Cell 2002, 110, 775-787.

5. Bae, J.H.; Schlessinger, J. Asymmetric tyrosine kinase arrangements in activation or autophosphorylation of receptor tyrosine kinases. Mol. Cells 2010, 29 443-448.

6. Chung, I.; Akita, R.; Vandlen, R.; Toomre, D.; Schlessinger, J.; Mellman, I. Spatial control of EGF receptor activation by reversible dimerization on living cells. Nature 2010, 464, 783-787.

7. Gan, H.K.; Kaye, A.H.; Luwor, R.B. The EGFRvIII variant in glioblastoma multiforme. J. Clin. Neurosci. 2009, 16 748-754.

8. Pedersen, M.W.; Meltorn, M.; Damstrup, L.; Poulsen, H. S. The type III epidermal growth factor receptor mutation. Biological significance and potential target for anti-cancer therapy. Ann. Oncol. 2001, 12, 745-760.

9. Johns, T.G.; Stockert, E.; Ritter, G.; Jungbluth, A.A.; Huang, H.J.; Cavenee, W.K.; Smyth, F.E.; Hall, C.M.; Watson, N.; Nice, E.C.; et al. Novel monoclonal antibody specific for the de2-7 epidermal growth factor receptor (EGFR) that also recognizes the EGFR expressed in cells containing amplification of the EGFR gene. Int. J. Cancer 2002, 98, 398-408.

10. Garrett, T.P.; Burgess, A.W.; Gan, H.K.; Luwor, R.B.; Cartwright, G.; Walker, F.; Orchard, S.G.; Clayton, A.H.; Nice, E.C.; Rothacker, J.; et al. Antibodies specifically targeting a locally misfolded region of tumor associated EGFR. Proc. Natl. Acad. Sci. USA 2009, 106, 5082-5087.

11. Johns, T.G.; Adams, T.E.; Cochran, J.R.; Hall, N.E.; Hoyne, P.A.; Olsen, M.J.; Kim, Y.S.; Rothacker, J.; Nice, E.C.; Walker, F.; et al. Identification of the epitope for the epidermal growth factor receptor-specific monoclonal antibody 806 reveals that it preferentially recognizes an untethered form of the receptor. J. Biol. Chem. 2004, 279, 30375-30384.

12. Lee, J.C.; Vivanco, I.; Beroukhim, R.; Huang, J.H.; Feng, W.L.; DeBiasi, R.M.; Yoshimoto, K.; King, J.C.; Nghiemphu, P.; Yuza, Y.; et al. Epidermal growth factor receptor activation in glioblastoma through novel missense mutations in the extracellular domain. PLoS Med. 2006, 3, e485.

13. The Cancer Genome Atlas Research Network. Comprehensive genomic characterization defines human glioblastoma genes and core pathways. Nature 2008, 455, 1061-1068. 
14. Huang, H.S.; Nagane, M.; Klingbeil, C.K.; Lin, H.; Nishikawa, R.; Ji, X.D.; Huang, C.M.; Gill, G.N.; Wiley, H.S.; Cavenee, W.K. The enhanced tumorigenic activity of a mutant epidermal growth factor receptor common in human cancers is mediated by threshold levels of constitutive tyrosine phosphorylation and unattenuated signaling. J. Biol. Chem. 1997, 272, 2927-2935.

15. Holbrook, M.R.; O’Donnell, J.B., Jr.; Slakey, L.L.; Gross, D.J. Epidermal growth factor receptor internalization rate is regulated by negative charges near the $\mathrm{SH} 2$ binding site $\mathrm{Tyr} 992$. Biochemistry 1999, 38, 9348-9356.

16. Fan, Q.W.; Cheng, C.; Knight, Z.A.; Haas-Kogan, D.; Stokoe, D.; James, C.D.; McCormick, F.; Shokat, K.M.; Weiss, W.A. EGFR signals to mTOR through PKC and independently of Akt in glioma. Sci. Signal. 2009, 2, ra4.

17. Huang, P.H.; Miraldi, E.R.; Xu, A.M.; Kundukulam, V.A.; Del Rosario, A.M.; Flynn, R.A.; Cavenee, W.K.; Furnari, F.B.; White, F.M. Phosphotyrosine signaling analysis of site-specific mutations on EGFRvIII identifies determinants governing glioblastoma cell growth. Mol. Biosyst. 2010, 6, 1227-1237.

18. Luwor, R.B.; Zhu, H.J.; Walker, F.; Vitali, A.A.; Perera, R.M.; Burgess, A.W.; Scott, A.M.; Johns, T.G. The tumor-specific de2-7 epidermal growth factor receptor (EGFR) promotes cells survival and heterodimerizes with the wild-type EGFR. Oncogene 2004, 23, 6095-6104.

19. Chu, C.T.; Everiss, K.D.; Wikstrand, C.J.; Batra, S.K.; Kung, H.J.; Bigner, D.D. Receptor dimerization is not a factor in the signalling activity of a transforming variant epidermal growth factor receptor (EGFRvIII). Biochem. J. 1997, 324, 855-861.

20. Moscatello, D.K.; Montgomery, R.B.; Sundareshan, P.; McDanel, H.; Wong, M.Y.; Wong, A.J. Transformational and altered signal transduction by a naturally occurring mutant EGF receptor. Oncogene 1996, 13, 85-96.

21. Fernandes, H.; Cohen, S.; Bishayee, S. Glycosylation-induced conformational modification positively regulates receptor-receptor association: A study with an aberrant epidermal growth factor receptor (EGFRvIII/DeltaEGFR) expressed in cancer cells. J. Biol. Chem. 2001, 276, 5375-5383.

22. Zhang, X.; Gureasko, J.; Shen, K.; Cole, P. A.; Kuriyan, J. An allosteric mechanism for activation of the kinase domain of epidermal growth factor receptor. Cell 2006, 125, 1137-1149.

23. Gan, H.K.; Walker, F.; Burgess, A.W.; Rigopoulos, A.; Scott, A.M.; Johns, T.G. The epidermal growth factor receptor (EGFR) tyrosine kinase inhibitor AG1478 increases the formation of inactive untethered EGFR dimers. Implications for combination therapy with monoclonal antibody 806. J. Biol. Chem. 2007, 282, 2840-2850.

24. Meierjohann, S.; Mueller, T.; Schartl, M.; Buehner, M. A structural model of the extracellular domain of the oncogenic EGFR variant Xmrk. Zebrafish 2006, 3, 59-69.

25. Robertson, S.C.; Meyer, A.N.; Hart, K.C.; Galvin, B.D.; Webster, M.K.; Donoghue, D.J. Activating mutations in the extracellular domain of the fibroblast growth factor receptor 2 function by disruption of the disulfide bond in the third immunoglobulin-like domain. Proc. Natl. Acad. Sci. USA 1998, 95, 4567-4572.

26. Watowich, S.S.; Yoshimura, A.; Longmore, G.D.; Hilton, D.J.; Yoshimura, Y.; Lodish, H.F. Homodimerization and constitutive activation of the erythropoietin receptor. Proc. Natl. Acad. Sci. USA 1992, 89, 2140-2144. 
27. Longmore, G.D.; Lodish, H.F. An activating mutation in the murine erythropoietin receptor induces erythroleukemia in mice: a cytokine receptor superfamily oncogene. Cell 1991, 67, 1089-1102.

28. Mangasarian, K.; Li, Y.; Mansukhani, A.; Basilico, C. Mutation associated with Crouzon syndrome causes ligand-independent dimerization and activation of FGF receptor-2. J. Cell. Physiol. 1997, 172, 117-125.

29. Santoro, M.; Carlomagno, F.; Romano, A.; Bottaro, D.P.; Dathan, N.A.; Grieco, M.; Fusco, A.; Vecchio, G.; Matoskova, B.; Kraus, M.H.; et al. Activation of RET as a dominant transforming gene by germline mutations of MEN2A and MEN2B. Science 1995, 267, 381-383.

30. Pillay, V.; Allaf, L.; Wilding, A.L.; Donoghue, J.F.; Court, N.W.; Greenall, S.A.; Scott, A.M.; Johns, T.G. The plasticity of oncogene addiction: implications for targeted therapies directed to receptor tyrosine kinases. Neoplasia 2009, 11, 448-458.

31. Siegel, P.M.; Muller, W.J. Mutations affecting conserved cysteine residues within the extracellular domain of Neu promote receptor dimerization and activation. Proc. Natl. Acad. Sci. USA 1996, 93, 8878-8883.

32. Prickett, T.D.; Agrawal, N.S.; Wei, X.; Yates, K.E.; Lin, J.C.; Wunderlich, J.R.; Cronin, J.C.; Cruz, P.; Rosenberg, S.A.; Samuels, Y. Analysis of the tyrosine kinome in melanoma reveals recurrent mutations in ERBB4. Nat. Genet. 2009, 41, 1127-1132.

(C) 2011 by the authors; licensee MDPI, Basel, Switzerland. This article is an open access article distributed under the terms and conditions of the Creative Commons Attribution license (http://creativecommons.org/licenses/by/3.0/). 